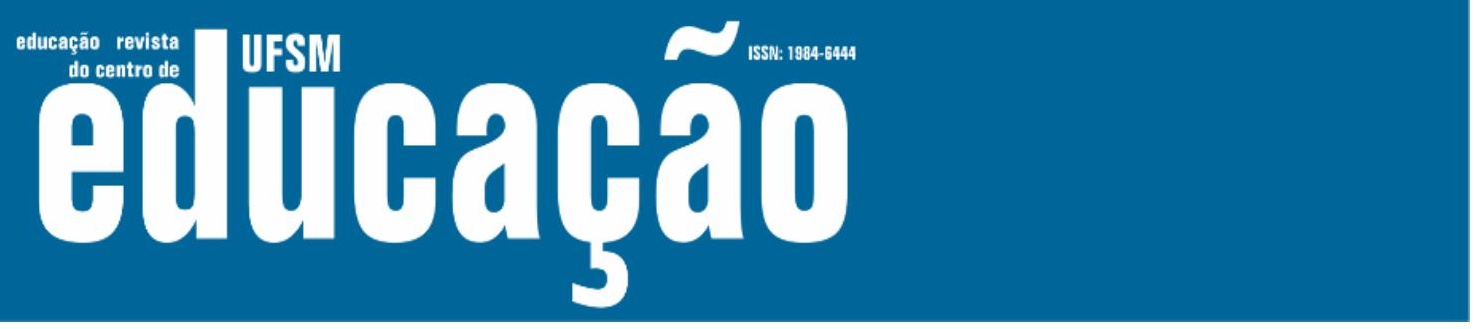

ISSN: 1984-6444 | http://dx.doi.org/10.5902/1984644440154

\title{
Clinique dialogique et rapport aux savoirs en situations extrêmes: à l'écoute des récits d'adolescentes malades
}

\author{
Dialogical clinic and relation to knowledge in extreme situations: listening \\ to the stories of sick teenagers
}

Clínica dialógica e relação aos saberes em situações extremas: à escuta dos relatos de adolescentes doentes

\author{
Martine Lani-Bayle \\ Professora doutora na Universidade de Nantes. Nantes, França. \\ martine.lani-bayle@dartybox.com - https://orcid.org/0000-0002-4549-8872 \\ Sandra Farias Maia-Vasconcelos \\ Professora doutora na Universidade Federal do Ceará. Fortaleza, Ceará, Brasil. \\ sandramaiafv@gmail.com - https://orcid.org/0000-0001-7201-6173
}

Recebido em 25 de setembro de 2019

Aprovado em 27 de janeiro de 2020

Publicado em 20 de março de 2020

\section{RESUMÉ}

Cette étude a pour but de savoir comment les adolescents hospitalisés ressentent leur vécu de malade chronique grave, hormis les maladies neurologiques sévères et les troubles psychologiques, et quels savoirs ils découvrent pendant la (les) période(s) d'hospitalisation. Cette recherche a travaillé auprès de cinq adolescents atteints de cancer, d'âges comprises entre 13 et 19 ans. Tous les adolescents ont été rencontrés à l'hôpital suivant la méthode de Conversations phénoménologiques (FERNANDES, 2017). La recherche a voulu questionner les différentes hypothèses possibles quant au rôle de la scolarisation pour l'adolescent gravement malade et son rapport au savoir en ce moment si difficile. Comme résultat, il a été vu qu'ils ont envie de parler d'euxmêmes, de tout ce qui leur arrive, de la maladie, des leurs sentiments, de leur manière de voir le monde et de tout ce à quoi ils n'avaient jamais pensé. Leur rapport à la classe hospitalière semble inaugurer un nouveau rapport aux savoirs scolaires, pas encore connu de l'école..

Mots-clés: Clinique dialogique; Rapport aux savoirs; Classe hospitalière.

\section{ABSTRACT}

The aim of this study is to find out how hospitalized teenagers feel about their experience as a serious chronic patient, except for severe neurological diseases and psychological disorders, and what knowledge they discover during the period of hospitalization. We worked with five teenagers with cancer of different etiologies, aged between 13 and 19 years old. All adolescents were met at the hospital following the 


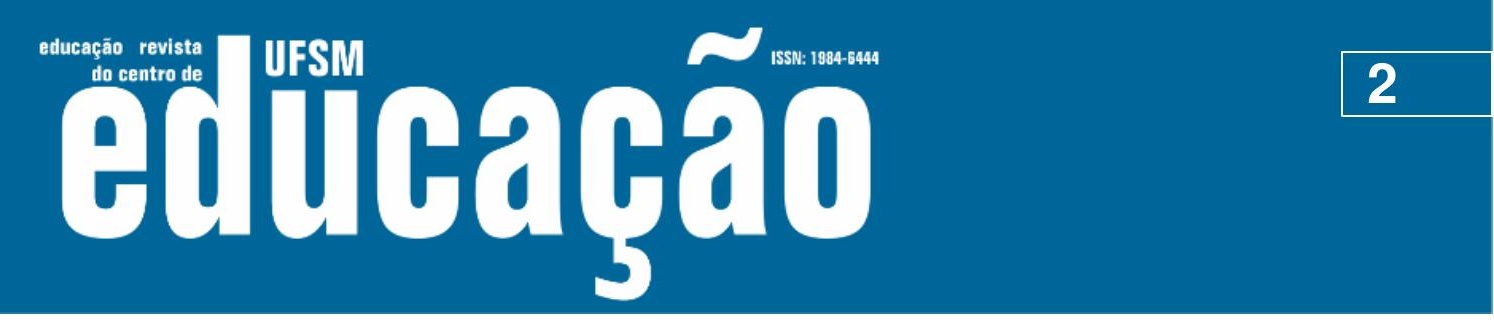

ISSN: 1984-6444 | http://dx.doi.org/10.5902/1984644440154

method of Phenomenological Conversations (FERNANDES, 2017). The objectif was to question the different possible hypotheses concerning the role of schooling for the severely ill teenager and his relationship to knowledge in this moment of physical difficulty and the suppression of the right to school. As a result, it has been realized that regular study is not needed for them, because what they want is to talk about themselves, about everything that happens to them, about their illness, their feelings, their way of seeing the world and everything about their lives. that they never thought. Its relationship with the hospital class seems to inaugurate a new relationship with school knowledge, not yet inaugurated by the school.

Keywords: Dialogical clinic; Relation to knowledge; Hospital class.

\section{RESUMO}

O objetivo deste estudo é conhecer como os adolescentes hospitalizados se sentem em relação à sua vivência como um paciente crônico grave, excetuando-se aqui os pacientes neurológicos severos e os com problemas psicológicos graves, e que saberes esses sujeitos desenvolvem durante o período de hospitalização. Trabalhouse com cinco adolescentes com cânceres de diferentes etiologias, com idades compreendidas entre 13 e 19 anos. Todos os adolescentes foram atendidos no hospital seguindo o método das Conversas Fenomenológicas (FERNANDES, 2017). O objetivo foi questionar as diferentes hipóteses possíveis sobre o papel da escolarização para o adolescente gravemente doente e sua relação com o saber neste momento de dificuldade física e de supressão do direito à escola. Como resultado, percebeu-se que o estudo regular não lhes faz falta, pois o que eles querem é falar sobre si mesmos, sobre tudo o que lhes acontece, sobre a doença, seus sentimentos, sua maneira de ver o mundo e tudo sobre o que nunca pensaram. Sua relação com a classe hospitalar parece inaugurar uma nova relação com o saber escolar, ainda não inaugurada pela escola.

Palavras-chave: Clínica Dialógica; Relação ao saber; Classe hospitalar.

\section{Introduction}

Le cancer est peut-être la maladie qui isole le plus souvent l'enfant de son école et de son milieu social. Pour essayer d'atténuer la perte scolaire, certains hôpitaux dans le monde, proposent des interventions scolaires aux individus hospitalisés. Nous ne resterons pas sur ce point (conf. Maia-Vasconcelos, 2015), notre objectif étant dans ce travail de donner une perception plus subjective, à savoir, nous nous inquiétons de savoir comment les adolescents ressentent leur vécu de malade chronique grave et quels savoirs ils découvrent pendant la (les) période(s) d'hospitalisation. Nous 


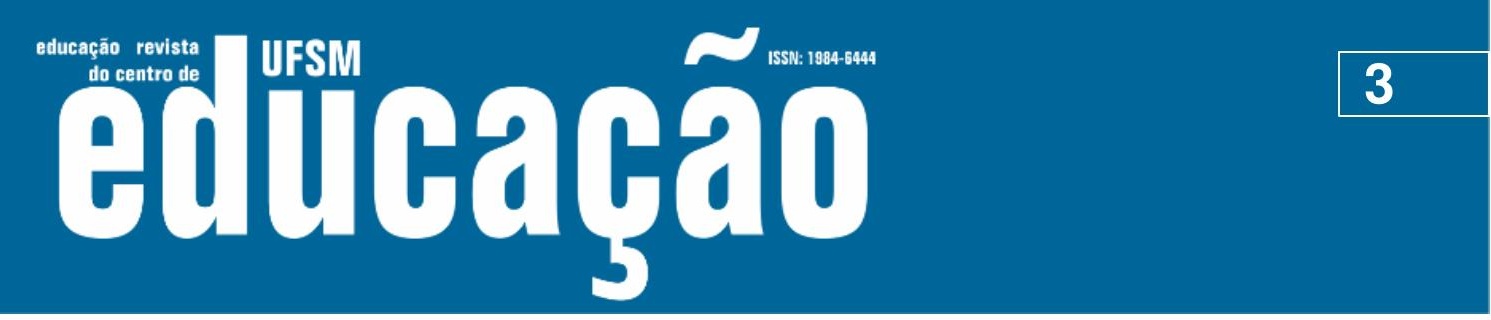

ISSN: 1984-6444 | http://dx.doi.org/10.5902/1984644440154

défendons que tout vécu devient savoir, même si le résultat de ce vécu n'est pas satisfaisant. Suivant la perception de Fikfak (2018), le vécu de tout individu est une expérience particulière dans la totalité de son corps. Nous pouvons élargir ce concept en affirmant que tout vécu est collectif, car cela touche toute l'humanité, même si à l'œil nu, parfois, nous ne nous centrons que sur les différences.

Dans cette étude, qui fait partie d'une recherche plus large ${ }^{1}$, nous avons focalisé sur les discours des adolescents atteints de cancer, hospitalisés, à propos de l'intervention scolaire proposée à l'hôpital. Parmi les adolescents hospitalisés dans le service de cet hôpital, nous avons choisi cinq adolescents. Cinq personnes. Cinq histoires vraies, cinq destins inconnus. C'est en ce sens que nous considérons que la problématique mérite d'être investiguée en s'appuyant sur des regards socio-éducatifs et sur la diversité des approches de sciences humaines.

Tous les adolescents ont été rencontrés à l'hôpital suivant la méthode de Conversations phénoménologiques (FERNANDES, 2017). C'est dans leurs réalités qui nous sommes allées. Leur parole et la nôtre étaient nos instruments de recherche. Le choix de cette stratégie s'appuie sur le fait que l'école est le milieu social le plus en évidence pour l'adolescent. Alors, nous avons voulu savoir comment cela se passe chez l'adolescent, questionner les hypothèses possibles quant au rôle de la scolarisation lorsqu'il est gravement malade et son rapport au savoir en ce moment si difficile.

\section{Enseignement hospitalier : l'expérience en situations extrêmes}

L'enseignement hospitalier est celui qui se réalise dans une institution de santé, clinique ou hôpital, et est dirigé aux individus qui ont subi une rupture dans leur formation. D’après Odile Delorme (2000 ; p. 35), «L'enseignement aux jeunes malades est plus qu'un apport de connaissances (...) est le garant de son équilibre et de son développement (...)». Fonseca $(2008 ; 2010)$ définit comme assistance scolaire à l'hôpital ou école hospitalière avec la même signification que celle donnée par l'agence de l'éducation nationale. 


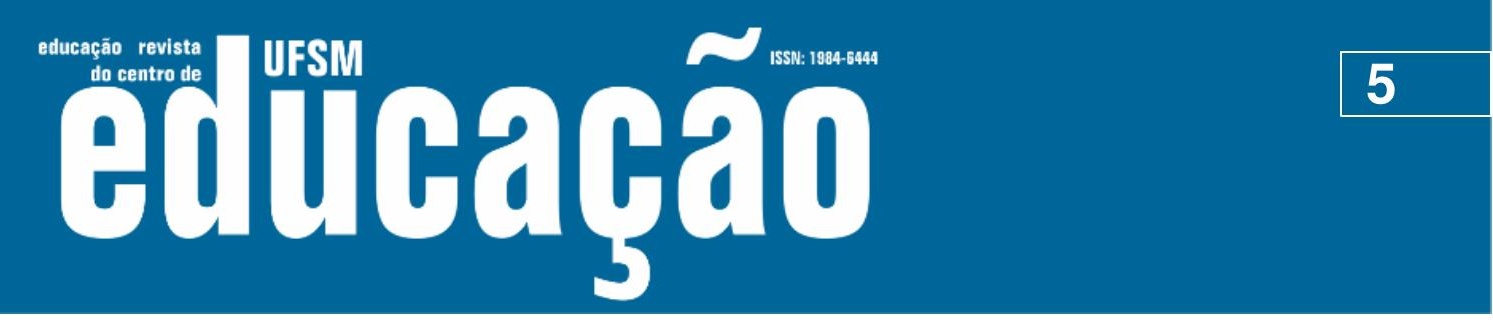

ISSN: 1984-6444 | http://dx.doi.org/10.5902/1984644440154

sensation de méconnaissance de soi. La solitude provoquée par l'éloignement de sa propre image comme un individu participant à des multiples activités est une des caractéristiques du cancer et cela provoque un sentiment d'étrangeté chez l'adolescent. S'il ne se reconnaît plus, comment reconnaître les autres et se faire reconnaître par les autres? Cette méconnaissance agit sur l'individu malade comme le manque de droit de savoir sur soi-même ce que les autres savent.

\section{La démarche dialogique pour connaître les sujets}

Les adolescents viennent de toutes régions du Ceará. Ils ont entre 14 et 19 ans, garçons et filles, mélangés malgré eux dans les chambres de cet hôpital. Chez tous, la même plainte: la vie et la scolarisation interrompue par la maladie, suivie de l'éloignement des copains, des interdictions diverses, du traitement très lourd et des préjugés de la société.

Depuis le début de la recherche, nous avons pu voir des scènes à ne plus oublier et entendre des paroles très significatives. Le lien formé avec ces personnes est rempli de subjectivité. Ils vivent un moment très spécial, vraiment inouï qui apporte des leçons très profondes sur la vie et sur la fragilité humaine. C'est pourquoi nous avons choisi l'approche narrative clinique, car cette approche révèle le sens de se mettre à l'écoute d'un sujet.

Comme il s'agit d'une démarche continue, il faut dire qu'après la présentation de notre projet aux adolescents et à leurs familles, les rencontres prennent un chemin particulier et individuel. Comme nous avons défendu dans des études antérieures (MAIA-VASCONCELOS, 2010) le vécu est un immense terrain où les apprentissages se réalisent, mais il doit être reconnu comme tel, sous le risque de rester oublié dans un coin infini de la mémoire, caché de tous et notamment de celui qui l'a vécu. Alors, dans notre rôle de chercheurs, nous voyons émerger chez chaque malade des histoires endormies, mais qui, alors réveillées, donnent forme aux réactions de ces personnes. C'est le début de la recherche de savoir et la découverte du rapport à ce savoir nouveau. 


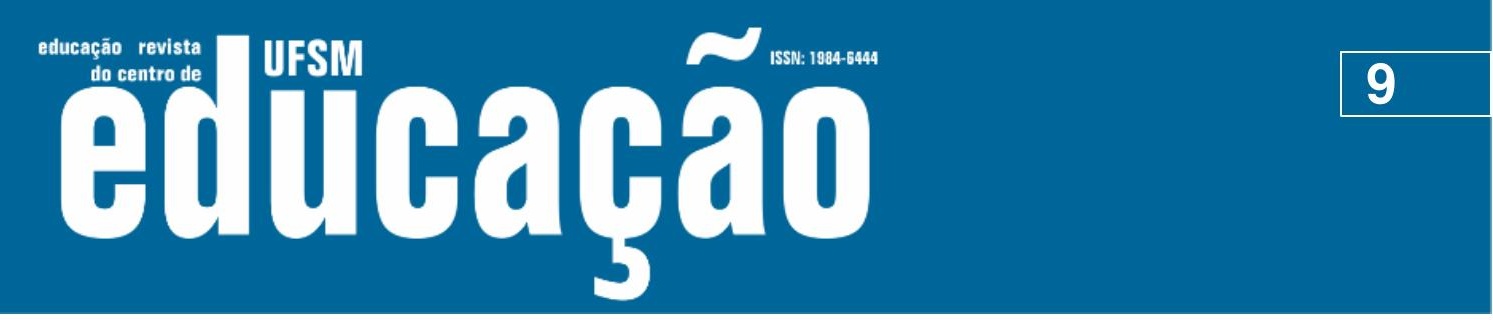

ISSN: 1984-6444 | http://dx.doi.org/10.5902/1984644440154

anodin, car toute situation de risque provoque l'incertitude, laquelle, d'après Paulo Freire (2001), est le seul lieu où il est possible de travailler les certitudes provisoires.

Nous allons maintenant voir comment le travail a été réalisé, par quels moyens et par quels chemins nous avons approché les adolescents hospitalisés et de quels apports théoriques nous nous sommes servies pour choisir les stratégies de travail.

\section{Méthodologie de travail : à la recherche des moyens de savoir}

Nos sujets ce sont des personnes, des mouvements, des pensées, des rêves. C'est pourquoi, avant de leur parler, il faut les voir, les observer dans leur pratique, en repérer les réactions et essayer de dénoyer leurs mystères. La recherche a donc poursuivi un chemin subjectif, mais un chemin nécessaire à la construction du sens dans les analyses qui l'ont succédé.

Le public étant hospitalisé, nous avons décidé de faire plusieurs rencontres brèves, plutôt que peu, mais longues. Le critère d'inclusion étant qu'ils soient en traitement depuis au moins un an. Lorsque le groupe s'est défini, en nombre de cinq personnes, nous avons fait un contrat de 10 rencontres conversationnelles, toutes enregistrées après accordance de la famille, d'environ une demi-heure chacune, cela pouvant changer à plus ou à moins, en fonction de l'état des adolescents et de leur présence à l'hôpital. Nous le présentons ici, en rappelant que tous les prénoms sont fictifs :

A1- Elisabeth a 14 ans. Diagnostiquée d'un Sarcome d'Ewing dans la jambe gauche et en traitement depuis l'âge de 12 ans. Elle a subi l'amputation radicale de la jambe l'année d'avant. Ses parents habitent dans la campagne et sa sœur l'accompagne constamment. Depuis le diagnostic, elle n'est jamais plus allée à l'école.

A2- Alain a 18 ans. Diagnostiqué de Leucémie LMA et est en traitement depuis l'âge de 16 ans. C'est un adolescent très éveillé et reste à l'hôpital avec sa mère. II aime la guitare et quoiqu'il ne soit jamais revenu à l'école, la guitare a toujours été sa compagne. 


\section{Fism oltibahá}

ISSN: 1984-6444 | http://dx.doi.org/10.5902/1984644440154

A3- René a 18 ans et est atteint d'un Sarcome d'Ewing du bras droit. II a subi l'amputation de son bras deux mois après le diagnostic. II vient d'une famille très pauvre de la campagne qui n'a pas de tradition scolaire. Ses parents sont analphabètes, travaillent comme agriculteurs, et le garçon les aidait depuis l'âge de 13 ans. II vient seul à l'hôpital.

A4- Juliette a 14 ans et souffre d'une Leucémie Myéloïde Aiguë depuis deux ans. Elle est la fille aînée d'une famille de trois enfants. Elle ne vient à l'hôpital jamais sans sa mère.

A5- Jessé a 19 ans et souffre d'un Rabdomyosarcome Para-testiculaire depuis deux ans et a subi l'ablation de ses organes sexuels. II est originaire d'une famille de classe moyenne. II est étudiant universitaire.

Des cinq personnes disponibles nous avons choisi de focaliser sur un pour approfondir l'étude, Jessé. Mais les autres ne resteront pas entièrement à l'écart. Cependant, avec Jessé, nous n'avons pas eu le temps de faire les dix rencontres, car il est mort dans la soirée du septième jour. Malgré cela, son discours nous a semblé le plus pertinents en ce qui concerne la problématique initiale de la recherche : la rupture du processus scolaire et de vie par une maladie, suivie de la formation d'un nouveau rapport au savoir.

Jessé n'a pas pu voir le texte transcrit, mais à chaque rencontre il réécoutait les extraits enregistrés, sans rien ajouter ou modifier. Comme cela suivait la méthodologie du départ, renforcé par la subjectivité qu'il a expressément voulu apporter, nous avons décidé de croiser son discours à celui des autres adolescents selon les catégories repérées.

\section{Le processus narratif: l'approche méthodologique de la construction du discours}

Nous avons ici l'objectif de montrer la place du discours narratif dans le processus de re-découverte du soi, de cette réflexion mûre et consciente sur la réalité immuable à présent. Pour aboutir à ce discours narratif, les rencontres 


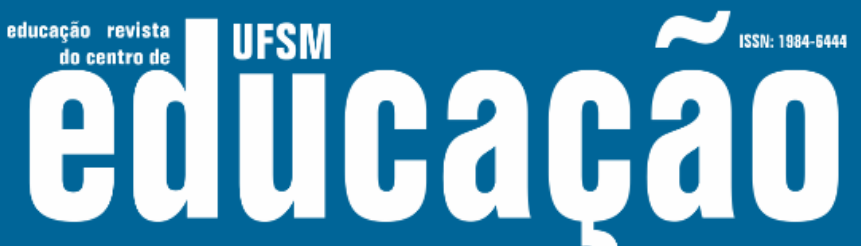

ISSN: 1984-6444 | http://dx.doi.org/10.5902/1984644440154

\section{Récits des adolescents}

Les premières rencontres ont donné la priorité aux sentiments concernant les conditions invariables des adolescents, à savoir : leur lieu de naissance, leurs parents, ce qu'ils faisaient avant le diagnostic, leur niveau scolaire, leurs histoires vécues, leur enfance, enfin, tout ce qui est dans leur passé et qui n'a pas été modifié par la maladie. Ce moment-là fait et réussi, c'est le moment du rapport qui survient, quand les adolescents nous font preuve de confiance. Avoir la confiance de ces adolescents exige que la rencontre ait une allure décontractée, comme une conversation entre amis, apparemment sans prétention scientifique.

C'est dans ce contexte qui s'est insérée la scolarisation et l'apport éducatif des enseignantes hospitalières et le rapport aux savoirs construits avant et pendant la maladie. La conscience de cette co-construction narrative par la conversation est une nouvelle manière apprise de prendre les gens au sens propre de vie.

Les conversations avec les sujets étaient individuelles et, à part le premier contact, ces rencontres ont été faites sans la présence de la mère. Le choix d'être seule avec les adolescents durant le temps de la conversation se justifie par deux facteurs : 1 - les adolescents se plaignent souvent de ce manque d'individualisation à I'hôpital ; 2- la présence de la mère pouvait gêner les adolescents au moment de parler. Nous avons choisi la conversation, parce que le récit oral permet l'émergence plus fidèle des sentiments, permet plus facilement la reconnaissance des tons dans les mots. Tout était important. La parole et le texte. Le dire, le dit et même le non-dit évident. Les sons et les tons. Les silences et les cris intérieurs présents dans les larmes parfois cachées par la gêne.

Les discours enregistrés, le travail de transcription était immédiat. Nous présentons ici un résumé de ces conversations sous forme de tableau selon les catégories suivantes 


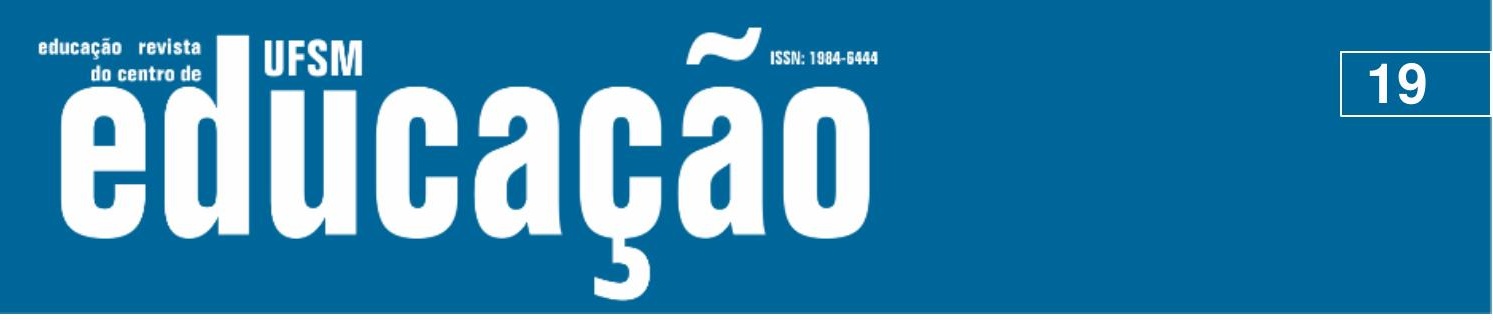

ISSN: 1984-6444 | http://dx.doi.org/10.5902/1984644440154

De GAULEJAC, Vincent. et ROY, S. Sociologies Cliniques, Epi - Desclée de Brouwer, 1993.

DELORME, Odile. (dir.) Etre malade et apprendre. Lyon : Chronique Sociale, 2000.

Dictionnaire étymologique et historique du Français. Paris : Larousse, 1964, 1993.

FERNANDES, Marcel Walline Carvalho Ferraz. Percepções fenomenológicas acerca da educação em turismo. Tese de doutorado. Doutorado em Turismo e Hotelaria, Universidade do Vale do Itajaí. Santa Catarina, Balneário Camburiú: 2017, 237 p.

FIKFAK, Jurij. "Entre la partie et le tout". Revue des sciences sociales [on-line], 60 | 2018, publicado em 15 de dezembro de 2018. Dsiponível em: http://journals.openedition.org/revss/1742 ; DOI : 10.4000/revss.1742. Acesso em: 21 mar. 2019.

FLACH, Fréderic. Resilience: how to bounce back when the going get tough!. New York: Hatherleigh Press, 1988.

FONSECA, Eneida Simões da. O lúdico no desenvolvimento e na aprendizagem da criança hospitalizada. In PÉREZ- RAMOS, Aidyl Queiroz; OLIVEIRA, Vera Barros de (Org.). Brincar é saúde: o lúdico como estratégia preventiva. Rio de Janeiro: WAK. 2010. p.203-224.

FONSECA, Eneida Simões da. Atendimento escolar no ambiente hospitalar. São Paulo: Memnon, 2008.

FREIRE, Paulo. A sombra desta mangueira. São Paulo : Olho d'Água, 4a. ed., 2001(a)

IMBERT, François. A propos de la démarche clinique. Cahier Pédagogique, $\mathrm{n}^{\circ} 234$, p. 148, mai 1995.

JOBIM e SOUZA, S. Infância e linguagem: Bakhtin, Vygotsky e Benjamin. Coleção Magistério. Campinas : Papirus, 3a ed.,1996.

LAINE, Alex. Faire de sa vie une histoire: Théories et pratiques de l'histoires de vie en formation. Paris : Desclée de Brouwer, 1998.

LANI-BAYLE, Martine. Les sciences de l'éducation en question. Recueil de Cahiers. 23, 29, 31 et 37. 2002.

MAGALHÃES, Izabel. Eu e tu: a constituição do sujeito no discurso médico. Brasília: Thesaurus, 2000, $181 \mathrm{p}$.

MAIA-VASCONCELOS, Sandra. Penser l'école et la construction des savoirs: une étude menée auprès d'adolescents cancéreux. 500f. Tese (Doutorado em Sciences de l'Éducation) - Université de Nantes, Nantes, 2003. 


\section{us:

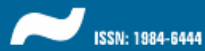

ISSN: 1984-6444 | http://dx.doi.org/10.5902/1984644440154

MAIA-VASCONCELOS, Sandra. Penser l'école et la construction des savoirs. Berlim: Editions Universitaires Européennes, 2010.

MAIA-VASCONCELOS, Sandra. Formação de professores para a Classe Hospitalar. Revista Educação Especial, v. 28, n. 51, jan./abr. 2015.

MONNERAY, Yves de la. La Parole rééducatrice: la relation d'aide à l'enfant en difficulté scolaire. Paris : Dunod, 1995.

PECHEUX, Michel. Discurso: estrutura et acontecimento. Illinois University Press. Trad. E. Orlandi. Campinas: Pontes, 1983.

SIBONY, Daniel. Entre-deux: l'origine en partage. Paris : Seuil, 1991

VILLIERS, Guy. La démarche clinique en sciences humaines. in: RENAULD d'ALLONES, C. et al. Histoire de vie comme méthode clinique. Paris: Dunod, 1989.

\section{Correspondência}

Martine Lani-Bayle - Université de Nantes - 1 Quai de Tourville, 44035 Nantes, França.

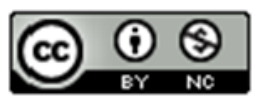

This work is licensed under a Creative Commons Attribution-NonCommercial 4.0 International (CC BY-NC 4.0)

\section{Notas}

\footnotetext{
${ }^{1}$ Estudo desenvolvido a partir de uma pesquisa guarda-chuva realizada na Universidade Federal do Ceará e registrada no CNPQ: Análise da construção do efeito de realidade e reflexividade nas narrativas infantis como estratégia de ensino de leitura 2016- em andamento.

${ }^{2}$ O projeto foi registrado e cadastrado no comitê de ética desde 1997, mas não havia então informatização no setor. Nunca obtivemos o registro formal, apenas o crachá que nos dava o direito de entrada ao setor de oncologia. A informatização teve início no ano 2000, com a mudança de direção e quando não pudemos mais atender nos leitos, mas somente nas salas de "recreação" do Bloco C.
} 